\title{
One Year Ago
}

National Cancer Institute

\section{Source}

National Cancer Institute. One Year Ago. NCI Thesaurus. Code C114880.

The time period one year before the current time. 